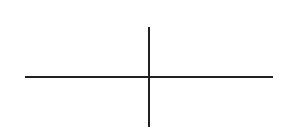

Tohoku Math. J.

57 (2005), 223-230

\title{
REAL HYPERSURFACES SOME OF WHOSE GEODESICS ARE PLANE CURVES IN NONFLAT COMPLEX SPACE FORMS
}

\author{
Dedicated to Professor Ryoichi Takagi on the occasion of his sixtieth birthday
}

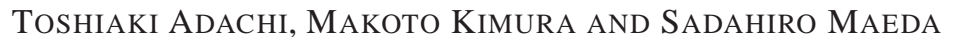

(Received July 23, 2003, revised April 1, 2004)

\begin{abstract}
In this paper we classify real hypersurfaces all of whose geodesics orthogonal to the characteristic vector field are plane curves in complex projective or complex hyperbolic spaces.
\end{abstract}

1. Introduction. Recent program of our study on submanifolds is based on the extrinsic shape of geodesics on submanifolds. From this point of view, a totally umbilic submanifold is considered to be the simplest one. It is well-known that a hypersurface $M^{n}$ in a real space form $\tilde{M}^{n+1}(c)$ of curvature $c$, which is one of a standard sphere $S^{n+1}(c)$, a Euclidean space $\boldsymbol{R}^{n+1}$ and a hyperbolic space $H^{n+1}(c)$, is totally umbilic if and only if every geodesic on this hypersurface $M^{n}$ is a plane curve in the ambient space $\tilde{M}^{n+1}(c)$. Here, a smooth curve on a Riemannian manifold $M$ is said to be a plane curve if it is locally contained on some real 2-dimensional totally geodesic submanifold of $M$. On the other hand, in an $n$-dimensional nonflat complex space form $\tilde{M}_{n}(c)$ of constant holomorphic sectional curvature $c$, which is either a complex projective space $\boldsymbol{C} P^{n}(c)$ or a complex hyperbolic space $\boldsymbol{C} H^{n}(c)$, there does not exist a real hypersurface $M^{2 n-1}$ all of whose geodesics are plane curves in the ambient space $\tilde{M}_{n}(c)$. This comes from the fact that a nonflat complex space form does not admit a totally umbilic real hypersurface. However, there exist real hypersurfaces $M^{2 n-1}$ all of whose geodesics orthogonal to the characteristic vector field $\xi$ of $M$ are plane curves in a nonflat complex space form. For example, every totally $\eta$-umbilic hypersurface has this property (see Section 2 for the definition of totally $\eta$-umbilic hypersurfaces).

Motivated by this geometric property of totally $\eta$-umbilic hypersurfaces, we are interested in classifying real hypersurfaces $M^{2 n-1}$ in a nonflat complex space form $\tilde{M}_{n}(c)$ all of whose geodesics orthogonal to the characteristic vector field $\xi$ of $M$ are plane curves in $\tilde{M}_{n}(c)$. The aim of this paper is to solve this problem:

2000 Mathematics Subject Classification. Primary 53B25; Secondary 53C40.

Key words and phrases. Real hypersurfaces, nonflat complex space forms, geodesics, plane curves, curves of order 2, totally $\eta$-umbilic, ruled real hypersurfaces.

The first author partially supported by Grant-in-Aid for Scientific Research (C) (No. 14540075), Japan Society for the Promotion of Science.

The second author partially supported by Grant-in-Aid for Scientific Research (C) (No. 15540075), Japan Society for the Promotion of Science.

The third author partially supported by Grant-in-Aid for Scientific Research (C) (No. 14540080), Japan Society for the Promotion of Science. . 


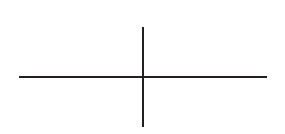

MAIN THEOREM. Let $M^{2 n-1}$ be a real hypersurface of a nonflat complex space form $\tilde{M}_{n}(c)(n \geqq 2)$. Then $M$ is locally congruent to a totally $\eta$-umbilic hypersurface or a ruled real hypersurface if and only if every geodesic $\gamma$ on $M$ whose initial vector $\dot{\gamma}(0)$ is orthogonal to the characteristic vector $\xi_{\gamma(0)}$ of $M$ is a plane curve in the ambient space $\tilde{M}_{n}(c)$.

We recall in Section 3 fundamental properties of curves of order 2 which are generalizations of plane curves. Also we review in Section 2 basic results on real hypersurfaces.

2. Basic results on real hypersurfaces. We shall start by summarizing some basic results on real hypersurfaces that will be useful in the following sections. Let $\tilde{M}_{n}(c)$ denote either a complex $n$-dimensional complex projective space $C P^{n}(c)$ of constant holomorphic sectional curvature $c$ or a complex $n$-dimensional complex hyperbolic space $\boldsymbol{C H}^{n}(c)$ of constant holomorphic sectional curvature $c$. Let $M^{2 n-1}$ be an orientable real hypersurface of $\tilde{M}_{n}(c)$ and $\mathcal{N}$ a unit normal vector field on $M$ in $\tilde{M}_{n}(c)$. The Riemannian connections $\tilde{\nabla}$ of $\tilde{M}_{n}(c)$ and $\nabla$ of $M$ are related by

$$
\tilde{\nabla}_{X} Y=\nabla_{X} Y+\langle A X, Y\rangle \mathcal{N} \text { and } \tilde{\nabla}_{X} \mathcal{N}=-A X
$$

for vector fields $X$ and $Y$ tangent to $M$, where $\langle$,$\rangle denotes the Riemannian metric on M$ induced from the standard metric on $\tilde{M}_{n}(c)$, and $A$ is the shape operator of $M$ in $\tilde{M}_{n}(c)$. It is known that $M$ admits an almost contact metric structure $(\phi, \xi, \eta,\langle\rangle$,$) induced from the$ Kähler structure $J$ of $\tilde{M}_{n}(c)$. The characteristic vector field $\xi$ of $M$ is defined as $\xi=-J \mathcal{N}$ and this structure satisfies

$$
\phi^{2}=-I+\eta \otimes \xi, \quad \eta(\xi)=1, \quad \text { and } \quad\langle\phi X, \phi Y\rangle=\langle X, Y\rangle-\eta(X) \eta(Y),
$$

where $I$ denotes the identity map of the tangent bundle $T M$ of $M$. It follows from the equalities (2.1) that

$$
\left(\nabla_{X} \phi\right) Y=\eta(Y) A X-\langle A X, Y\rangle \xi
$$

and

$$
\nabla_{X} \xi=\phi A X .
$$

The eigenvalues and eigenvectors of the shape operator $A$ are called principal curvatures and principal curvature vectors, respectively. The condition that the characteristic vector $\xi=$ $-J \mathcal{N}$ is principal is quite natural. As was shown in [NR], for a real hypersurface $M^{2 n-1}$ in $\tilde{M}_{n}(c)(n \geqq 2)$, if $A \xi=\delta \xi$ holds with some function $\delta$ on $M$ then $\delta$ is locally constant. In $C P^{n}$, each real hypersurface $M$ lying on a tube of constant radius $r(>0)$ around a complex submanifold of $\boldsymbol{C} P^{n}$ satisfies this condition on $\xi$. In $\boldsymbol{C} H^{n}$, each real hypersurface $M$ lying on a tube around a complex submanifold or around a totally real submanifold of $\boldsymbol{C H}^{n}$ satisfies that condition. We usually call $M$ a Hopf hypersurface, when the characteristic vector $\xi$ is principal.

A real hypersurface $M$ of $\tilde{M}_{n}(c)(n \geqq 2)$ is called a totally $\eta$-umbilic, if its shape operator $A$ is of the form $A=\alpha I+\beta \eta \otimes \xi$ for some functions $\alpha$ and $\beta$ on $M$. It is known that every totally $\eta$-umbilic hypersurface is a typical example of a Hopf hypersurface. The following 


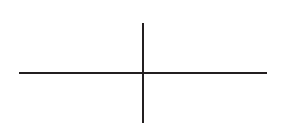

classification theorem of totally $\eta$-umbilic hypersurfaces $M$ shows that these two functions $\alpha$ and $\beta$ are automatically constant on $M$ (see [NR]):

THEOREM A. A totally $\eta$-umbilic hypersurface $M^{2 n-1}$ of a nonflat complex space form $\tilde{M}_{n}(c)(n \geqq 2)$ is locally congruent to one of the following:

(P) geodesic spheres of radius $r(0<r<\pi / \sqrt{c})$ in $\boldsymbol{C} P^{n}(c)$,

(H) i) horospheres in $\mathrm{CH}^{n}(c)$,

ii) geodesic spheres of radius $r(0<r<\infty)$ in $\boldsymbol{C} H^{n}(c)$,

iii) tubes of radius $r(0<r<\infty)$ around totally geodesic complex hyperplane $\mathrm{CH}^{n-1}(c)$ in $\mathrm{CH}^{n}(c)$.

It is known that every totally $\eta$-umbilic hypersurface $M$ has two distinct constant principal curvatures and satisfies the condition that the structure tensor $\phi$ and the shape operator $A$ of $M$ in $\tilde{M}_{n}(c)$ are commutative: $\phi A=A \phi$.

Next we recall ruled real hypersurfaces in a nonflat complex space form, which are typical examples of non-Hopf hypersurfaces. A real hypersurface $M$ is called a ruled real hypersurface of a nonflat complex space form $\tilde{M}_{n}(c)(n \geqq 2)$ if the holomorphic distribution $T^{0}$ defined by $T^{0}(x)=\left\{X \in T_{x} M \mid X \perp \xi\right\}$ for $x \in M$ is integrable and each of its integral manifolds is a totally geodesic complex hypersurface $M_{n-1}(c)$ of $\tilde{M}_{n}(c)$. A ruled real hypersurface is constructed in the following manner. Given an arbitrary regular curve $\gamma$ defined on an interval $I$ in $\tilde{M}_{n}(c)$ we have at each point $\gamma(t)(t \in I)$ a totally geodesic complex hypersurface $M_{n-1}^{(t)}(c)$ that is orthogonal to the plane spanned by $\{\dot{\gamma}(t), J \dot{\gamma}(t)\}$. Then we see that $M=\bigcup_{t \in I} M_{n-1}^{(t)}(c)$ is a ruled real hypersurface in $\tilde{M}_{n}(c)$. The following gives a characterization of ruled real hypersurfaces in terms of the shape operator $A$.

Proposition B (see [NR]). Let $M$ be a real hypersurface in a nonflat complex space form $\tilde{M}_{n}(c)(n \geqq 2)$. Then the following conditions are equivalent:

(1) $M$ is a ruled real hypersurface.

(2) The shape operator $A$ of $M$ satisfies the following equalities with a unit vector $U$ orthogonal to $\xi$ :

$$
A \xi=\mu \xi+\nu U, \quad A U=v \xi, \quad A X=0,
$$

where $\mu, v$ are differentiable functions on $M$ with $v \neq 0$, and $X$ is an arbitrary tangent vector orthogonal to $\xi$ and $U$.

(3) The shape operator A of $M$ satisfies $\langle A X, Y\rangle=0$ for any tangent vectors $X, Y \in$ $T_{x} M$ orthogonal to $\xi$ at each point $x \in M$.

3. Curves of order 2. A smooth curve $\gamma$ on a complete Riemannian manifold $M$ parametrized by its arclength is called a curve of order 2 if it satisfies the following nonlinear differential equation:

$$
\left\|\nabla_{\dot{\gamma}} \dot{\gamma}\right\|^{2}\left\{\nabla_{\dot{\gamma}} \nabla_{\dot{\gamma}} \dot{\gamma}+\left\|\nabla_{\dot{\gamma}} \dot{\gamma}\right\|^{2} \dot{\gamma}\right\}=\left\langle\nabla_{\dot{\gamma}} \dot{\gamma}, \nabla_{\dot{\gamma}} \nabla_{\dot{\gamma}} \dot{\gamma}\right\rangle \nabla_{\dot{\gamma}} \dot{\gamma},
$$

where $\nabla_{\dot{\gamma}}$ denotes the covariant differentiation along $\gamma$ with respect to the Riemannian connection $\nabla$ on $M$. This is a generalization of the notion of circles. We call a smooth curve $\gamma$ 


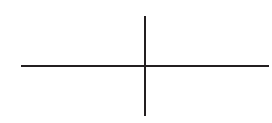

parametrized by its arclength a circle if it satisfies $\nabla_{\dot{\gamma}} \nabla_{\dot{\gamma}} \dot{\gamma}=-k^{2} \dot{\gamma}$ with some nonnegative constant $k$. This condition is equivalent to the condition that there exist a nonnegative constant $k$ and a field of unit vectors $Y$ along this curve that satisfy $\nabla_{\dot{\gamma}} \dot{\gamma}=k Y$ and $\nabla_{\dot{\gamma}} Y=-k \dot{\gamma}$. Since $k=\left\|\nabla_{\dot{\gamma}} \dot{\gamma}\right\|$, we see that circles are curves of order 2 . We call this constant $k$ the curvature of $\gamma$. We note that geodesics are treated as circles of null curvature. On a real space form $M^{n}(c)$ circles are plane curves, but it is not true in general. For a circle $\gamma$ on a complex projective space we have an important invariant $\tau=\left\langle\dot{\gamma}, J \nabla_{\dot{\gamma}} \dot{\gamma}\right\rangle /\left\|\nabla_{\dot{\gamma}} \dot{\gamma}\right\|$, which is called the complex torsion. When $\tau= \pm 1$ it lies on a totally geodesic complex projective line, and when $\tau=0$ it lies on a totally geodesic real projective plane. However in the case $\tau \neq 0, \pm 1$ it does not lie on any totally geodesic real 2-dimensional submanifold (see [AMU]). The same result holds for a circle on a complex hyperbolic space (see [AM1]).

A smooth curve $\gamma=\gamma(s)$ parametrized by its arclength $s$ is called a Frenet curve of proper order 2 if there exist a smooth unit vector field $Y$ along $\gamma$ orthogonal to $\dot{\gamma}$ and a smooth positive function $\kappa$ satisfying

$$
\nabla_{\dot{\gamma}} \dot{\gamma}(s)=\kappa(s) Y(s) \quad \text { and } \quad \nabla_{\dot{\gamma}} Y(s)=-\kappa(s) \dot{\gamma}(s) .
$$

The function $\kappa$ and the orthonormal frame $\{\dot{\gamma}, Y\}$ are called its curvature and Frenet frame, respectively. Trivially a circle of positive curvature is a Frenet curve of proper order 2 . We call a curve a Frenet curve of order 2 if it is either a geodesic or a Frenet curve of proper order 2. These two notions are related as follows.

LEMMA 1. (1) Every Frenet curve of order 2 is a curve of order 2.

(2) If a curve $\gamma$ of order 2 satisfies $\left\|\nabla_{\dot{\gamma}} \dot{\gamma}(s)\right\|>0$ for all $s$, then it is a Frenet curve of proper order 2, whose curvature and Frenet frame are $\kappa(s)=\left\|\nabla_{\dot{\gamma}} \dot{\gamma}(s)\right\|$ and $\{\dot{\gamma}, Y=$ $\left.\nabla_{\dot{\gamma}} \dot{\gamma} /\left\|\nabla_{\dot{\gamma}} \dot{\gamma}\right\|\right\}$, respectively.

PROOF. We can get the first assertion by direct computation. To show the second we put $\kappa(s)=\left\|\nabla_{\dot{\gamma}} \dot{\gamma}(s)\right\|$. As we have $\kappa \kappa^{\prime}=\left\langle\nabla_{\dot{\gamma}} \dot{\gamma}, \nabla_{\dot{\gamma}} \nabla_{\dot{\gamma}} \dot{\gamma}\right\rangle$, the equation (3.1) guarantees that the vector field $Y=(1 / \kappa) \nabla_{\dot{\gamma}} \dot{\gamma}$ satisfies

$$
\nabla_{\dot{\gamma}} Y=\frac{1}{\kappa^{3}}\left(\kappa^{2} \nabla_{\dot{\gamma}} \nabla_{\dot{\gamma}} \dot{\gamma}-\kappa \kappa^{\prime} \nabla_{\dot{\gamma}} \dot{\gamma}\right)=-\kappa \dot{\gamma},
$$

which leads us to the conclusion.

It should be noted that we allow a curve $\gamma$ of order 2 to have points where $\nabla_{\dot{\gamma}} \dot{\gamma}$ vanishes. For a curve of order 2 we call such a point an inflection point. One can easily find that a cubic curve $y=x^{3}$ on a Euclidean $x y$-plane has an inflection point at the origin. We have to take care of inflection points. The following lemma tells us that the notion of curves of order 2 is also an extension of the notion of plane curves.

LEMMA 2. Every smooth plane curve parametrized by its arclength is also a curve of order 2.

PROOF. Let $\gamma$ be a smooth plane curve parametrized by its arclength. For each $s_{0}$ we have a real 2-dimensional totally geodesic submanifold $S$ and positive number $\delta$ such that 


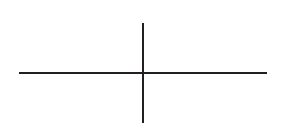

REAL HYPERSURFACES IN NONFLAT COMPLEX SPACE FORMS

the restriction $\left.\gamma\right|_{\left(s_{0}-\delta, s_{0}+\delta\right)}$ lies on $S$. We then have a local smooth unit vector field $V$ along $\left.\gamma\right|_{\left(s_{0}-\delta, s_{0}+\delta\right)}$ that is orthogonal to $\dot{\gamma}$ and is tangent to $S$. Since $\|\dot{\gamma}\|=\|V\|=1$, we see $\langle\dot{\gamma}, V\rangle=\left\langle V, \nabla_{\dot{\gamma}} V\right\rangle=0$. Since $S$ is 2-dimensional and totally geodesic, we see that $\nabla_{\dot{\gamma}} \dot{\gamma}$ is proportional to $V$ and $\nabla_{\dot{\gamma}} V$ is proportional to $\dot{\gamma}$. By differentiating $\langle\dot{\gamma}, V\rangle=0$, we get $\nabla_{\dot{\gamma}} \dot{\gamma}=v V$ and $\nabla_{\dot{\gamma}} V=-v \dot{\gamma}$ with a function $\nu$.

However, in general, a curve of order 2 is not necessarily a plane curve (see [SMA] for example).

4. Proof of the main theorem. We are now in a position to prove our main theorem. We here show a bit more detailed result.

THEOREM. Let $M^{2 n-1}$ be a real hypersurface of a nonflat complex space form $\tilde{M}_{n}(c)(n \geqq 2)$. Then the following conditions are equivalent:

(1) $M$ is locally congruent to either a totally $\eta$-umbilic hypersurface or a ruled real hypersurface.

(2) Every geodesic $\gamma$ on $M$ whose initial vector $\dot{\gamma}(0)$ is orthogonal to the characteristic vector $\xi_{\gamma(0)}$ is a plane curve in the ambient space $\tilde{M}_{n}(c)$.

(3) Every geodesic $\gamma$ on $M$ whose initial vector $\dot{\gamma}(0)$ is orthogonal to the characteristic vector $\xi_{\gamma(0)}$ is a circle in the ambient space $\tilde{M}_{n}(c)$.

(4) Every geodesic $\gamma$ on $M$ whose initial vector $\dot{\gamma}(0)$ is orthogonal to the characteristic vector $\xi_{\gamma(0)}$ is a curve of order 2 in the ambient space $\tilde{M}_{n}(c)$.

PROOF. It is trivial that the second condition implies the fourth condition and the third condition also implies the fourth.

We first show that the first condition yields the second and the third. Let $M$ be a totally $\eta$-umbilic hypersurface in $\tilde{M}_{n}(c)$. Then all tangent vectors orthogonal to $\xi$ are principal curvature vectors with a single principal curvature; that is, there is a constant $\lambda$ with $A u=\lambda u$ for each tangent vector $u \in T M$ orthogonal to $\xi$. The principal curvature is

$$
\lambda= \begin{cases}(\sqrt{c} / 2) \cot \sqrt{c} r / 2, & \text { a geodesic sphere } M \text { of radius } r \text { in } \boldsymbol{C} P^{n}(c), \\ (\sqrt{|c|} / 2) \operatorname{coth} \sqrt{|c|} r / 2, & \text { a geodesic sphere } M \text { of radius } r \text { in } \boldsymbol{C} H^{n}(c), \\ (\sqrt{|c|} / 2) \tanh \sqrt{|c|} r / 2, & \text { a tube } M \text { of radius } r \text { around totally } \\ & \text { geodesic } \boldsymbol{C} H^{n-1}(c) \text { in } \boldsymbol{C} H^{n}(c), \\ (\sqrt{|c|} / 2), & \text { a horosphere } M \text { in } \boldsymbol{C} H^{n}(c) .\end{cases}
$$

Let $\gamma=\gamma(s)$ be a geodesic on $M$ with initial vector $\dot{\gamma}(0)$ orthogonal to $\xi_{\gamma(0)}$. By (2.3) and the equality $\phi A=A \phi$, we see that

$$
\dot{\gamma}\langle\dot{\gamma}, \xi\rangle=\left\langle\nabla_{\dot{\gamma}} \dot{\gamma}, \xi\right\rangle+\left\langle\dot{\gamma}, \nabla_{\dot{\gamma}} \xi\right\rangle=\langle\dot{\gamma}, \phi A \dot{\gamma}\rangle=\langle\dot{\gamma}, A \phi \dot{\gamma}\rangle=-\langle\phi A \dot{\gamma}, \dot{\gamma}\rangle=0 .
$$

Therefore the tangential vector $\dot{\gamma}(s)$ is perpendicular to the characteristic vector $\xi$ at each $s$. This, together with the equalities (2.1), implies $\tilde{\nabla}_{\dot{\gamma}} \dot{\gamma}=\lambda \mathcal{N}$ and $\tilde{\nabla}_{\dot{\gamma}} \mathcal{N}=-\lambda \dot{\gamma}$. Hence the curve $\gamma$ is a circle of positive curvature $\lambda$ in the ambient space $\tilde{M}_{n}(c)$ with complex torsion $\tau=\langle\dot{\gamma}, J \mathcal{N}\rangle=0$. So, when $c>0$, this curve $\gamma$ lies on a 2-dimensional real projective 


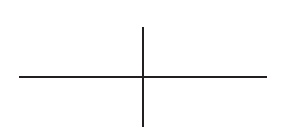

space $\boldsymbol{R} P^{2}(c / 4)$ of curvature $c / 4$ which is totally real totally geodesic in $\boldsymbol{C} P^{n}(c)$, and when $c<0$, this curve $\gamma$ lies on a 2-dimensional hyperbolic space $H^{2}(c / 4)$ of curvature $c / 4$ that is totally real totally geodesic in $\boldsymbol{C H}(c)$. Thus we could check the assertion in the case of totally $\eta$-umbilic hypersurfaces.

Next, we shall check the assertion in the case of ruled real hypersurfaces $M$. Let $\gamma$ be a geodesic on $M$ with initial vector $\dot{\gamma}(0)$ orthogonal to $\xi_{\gamma(0)}$. Let $M_{n-1}(c)$ be the integral manifold through the point $x=\gamma(0)$ for the holomorphic distribution $T^{0}$. Since $\dot{\gamma}(0) \in$ $T_{x} M_{n-1}(c)$ and $M_{n-1}(c)$ is a totally geodesic complex hypersurface of the ambient space $\tilde{M}_{n}(c)$, we see that $\gamma$ locally lies on $M_{n-1}(c)$ by the uniqueness theorem on geodesics. Hence the geodesic $\gamma$ is a geodesic in $\tilde{M}_{n}(c)$. In particular, since the ambient space $\tilde{M}_{n}(c)$ is a rank one symmetric space, $\gamma$ is a plane curve in $\tilde{M}_{n}(c)$.

It remains to verify that the fourth condition implies the first. Let $M$ be a real hypersurface in $\tilde{M}_{n}(c)$ satisfying the fourth condition. We take an orthonormal pair of tangent vectors $u, v$ at an arbitrary point $x \in M$ that are perpendicular to $\xi$. We first show

$$
\langle A u, u\rangle\langle A u, v\rangle=0 .
$$

We here suppose that $\langle A u, u\rangle \neq 0$. Let $\gamma=\gamma(s)$ be a geodesic on $M$ with the initial condition that $\gamma(0)=x$ and $\dot{\gamma}(0)=u$. There exists some $\varepsilon>0$ satisfying that $\langle A \dot{\gamma}(s), \dot{\gamma}(s)\rangle \neq 0$ for each $s(-\varepsilon<s<\varepsilon)$. By the first equality in (2.1), this shows that $\left\|\tilde{\nabla}_{\dot{\gamma}} \dot{\gamma}(s)\right\|>0$ for each $s(-\varepsilon<s<\varepsilon)$. Hence Lemma 1 tells us that the curve $\gamma$ in $\tilde{M}_{n}(c)$ restricted to the interval $-\varepsilon<s<\varepsilon$ is a Frenet curve of proper order 2. Without loss of generality we may consider the case $\langle A \dot{\gamma}(s), \dot{\gamma}(s)\rangle>0$ for each $s(-\varepsilon<s<\varepsilon)$. Then the curvature and Frenet frame of $\gamma$ in $\tilde{M}_{n}(c)$ are $\kappa(s)=\langle A \dot{\gamma}(s), \dot{\gamma}(s)\rangle$ and $\{\dot{\gamma}, \mathcal{N}\}$, respectively, so that it satisfies $\tilde{\nabla}_{\dot{\gamma}} \dot{\gamma}=\kappa(s) \mathcal{N}$ and $\tilde{\nabla}_{\dot{\gamma}} \mathcal{N}=-\kappa(s) \dot{\gamma}$. This, combined with the second equality in (2.1), shows that $A \dot{\gamma}(s)=\kappa(s) \dot{\gamma}(s)$ for each $s(-\varepsilon<s<\varepsilon)$, and in particular shows that $A u=k u$ with a positive $k=\langle A u, u\rangle$. Thus we have $\langle A u, v\rangle=0$, and get (4.1) for each orthonormal pair of vectors $u, v$ orthogonal to $\xi$.

Now let $f: S^{2 n-3}(1)\left(\subset \boldsymbol{R}^{2 n-2}\right) \rightarrow \boldsymbol{R}$ be the differentiable function on a subset $S^{2 n-3}(1) \cong\left\{u \in U_{x} M \mid u \perp \xi_{x}\right\}$ of the unit tangent space $U_{x} M$ defined by $f(u)=\langle A u, u\rangle^{2}$, where $A$ is the shape operator of $M$ at the point $x$. If $v$ is a vector tangent to $S^{2 n-3}(1)$ at $u$ (hence $u \perp v$ ), we find $v(f)=4\langle A u, u\rangle\langle A u, v\rangle=0$ by (4.1). Thus $f$ is a constant function, that is,

$$
|\langle A u, u\rangle|=k \quad \text { for each unit vector } u \text { orthogonal to } \xi_{x},
$$

with some nonnegative constant $k=k(x)$.

When $k(x) \neq 0$, by virtue of the above discussion we see that $A u=k u$ or $A u=-k u$ for each $u$. Then we easily find out that either $A u=k u$ for all $u \in T_{x} M$ orthogonal to $\xi_{x}$ or $A u=-k u$ for all $u \in T_{x} M$ orthogonal to $\xi_{x}$. This tells us that $x$ is an $\eta$-umbilic point of our real hypersurface $M$ of $\tilde{M}_{n}(c)$ and the function $k=k(x)$ is locally constant on some sufficiently small neighborhood $\mathcal{U}$ of the point $x$, so that $M$ is congruent to one of the totally $\eta$-umbilic hypersurfaces in the neighborhood $\mathcal{U}$. 


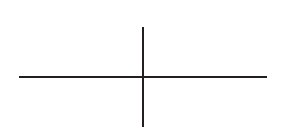

REAL HYPERSURFACES IN NONFLAT COMPLEX SPACE FORMS

When $k(x)=0$ it follows from the continuity of the function $k=k(x)$ and the above argument that there exists some neighborhood $\mathcal{V}$ of $x$ satisfying $k \equiv 0$ on $\mathcal{V}$. Thus we find that $\langle A u, u\rangle=0$ for all $u \in U_{y} M$ orthogonal to $\xi_{y}$ at an arbitrary point $y$ on $\mathcal{V}$. This, together with the fact that $A$ is symmetric and from Proposition $\mathrm{B}$, implies that the real hypersurface $M$ is congruent to a ruled real hypersurface on $\mathcal{V}$.

REMARK. By the proof of Theorem we see that each geodesic orthogonal to $\xi$ on a ruled real hypersurface $M$ in $\tilde{M}_{n}(c)(n \geqq 2)$ is a geodesic on $\tilde{M}_{n}(c)$. When $M$ is a totally $\eta$-umbilic hypersurface in $\tilde{M}_{n}(c)$, each geodesic orthogonal to $\xi$ is a circle on the ambient space $\tilde{M}_{n}(c)$ with the following curvature:

$$
\begin{cases}(\sqrt{c} / 2) \cot \sqrt{c} r / 2, & M \text { is a geodesic sphere of radius } r \text { in } \boldsymbol{C} P^{n}(c), \\ (\sqrt{|c|} / 2) \operatorname{coth} \sqrt{|c|} r / 2, & M \text { is a geodesic sphere of radius } r \text { in } \boldsymbol{C} H^{n}(c), \\ (\sqrt{|c|} / 2) \tanh \sqrt{|c|} r / 2, & M \text { is a tube of radius } r \text { around totally } \\ & \text { geodesic } \boldsymbol{C} H^{n-1}(c) \text { in } \boldsymbol{C} H^{n}(c), \\ (\sqrt{|c|} / 2), & M \text { is a horosphere in } \boldsymbol{C} H^{n}(c) .\end{cases}
$$

Finally we should remark that the following theorem is closely related to our result.

THEOREM C (see [AMY]). A real hypersurface $M^{2 n-1}$ in a nonflat complex space form $\tilde{M}_{n}(c)(n \geqq 2)$ is locally congruent to one of the totally $\eta$-umbilic hypersurfaces if and only if at each point $x \in M$ there exist orthonormal tangent vectors $v_{1}, v_{2}, \ldots, v_{2 n-2} \in T_{x} M$ orthogonal to the characteristic vector $\xi$ such that all geodesics on $M$ through $x$ in the direction $v_{i}+v_{j}(1 \leqq i \leqq j \leqq 2 n-2)$ are circles of positive curvatures in the ambient space $\tilde{M}_{n}(c)$.

In the statement of Theorem C, if we replace "circles of positive curvatures" by "circles", then this theorem is no longer true. For example let us recall ruled real hypersurfaces. Here is anther example worth mentioning:

EXAMPLE. Let $M$ be a tube of radius $\pi / 4$ over totally geodesic $C P^{k}(4)(1 \leqq k \leqq n-2)$ in $C P^{n}(4)$. It is known that $T^{0} M=V_{-1} \oplus V_{1}$, where $V_{-1}=\{X \in T M \mid A X=-X\}$ and $V_{1}=\{X \in T M \mid A X=X\}$, with $\operatorname{dim} V_{-1}=2 k$ and $\operatorname{dim} V_{1}=2 n-2-2 k$, respectively. Take orthonormal vectors $v_{1}, v_{2}, \ldots, v_{2 n-2}$ orthogonal to $\xi$ at an arbitrary point $x$ of $M$ in such a way that $v_{j} \in V_{-1}$ for $1 \leqq j \leqq 2 k$ and $v_{j} \in V_{1}$ for $2 k+1 \leqq j \leqq 2 n-2$. Then all geodesics of $M$ through $x$ in the direction $v_{i}+v_{j}(1 \leqq i \leqq j \leqq 2 n-2)$ are circles in $\boldsymbol{C} P^{n}(4)$. However, it is needless to say that this real hypersurface $M$ is neither a totally $\eta$-umbilic hypersurface nor a ruled real hypersurface in $C P^{n}(4)$. We particularly note that all geodesics of $M$ through $x$ in the direction $v_{i}+v_{j}(1 \leqq i \leqq 2 k, 2 k+1 \leqq j \leqq 2 n-2)$ are also geodesics in the ambient space $\boldsymbol{C} P^{n}(4)$. 


\section{REFERENCES}

[AM1] T. ADACHI AND S. MAEDA, Global behaviours of circles in a complex hyperbolic space, Tsukuba J. Math. 21 (1997), 29-42.

[AM2] T. AdACHI AND S. MAEDA, Characterizations of space forms by circles on their geodesic spheres, Proc. Japan Acad. Ser. A Math. Sci. 78 (2002), 143-147.

[AMU] T. ADACHI, S. MAEDA AND S. UdAGaWA, Circles in a complex projective space, Osaka J. Math. 32 (1995), 709-719.

[AMY] T. ADACHI, S. MAEDA AND M. YAMAGISHI, Length spectrum of geodesic spheres in a non-flat complex space form, J. Math. Soc. Japan 54 (2002), 373-408.

[NR] R. NiEbERGaLl AND P. J. RYAN, Real hypersurfaces in complex space forms, Tight and taut submanifolds (Berkeley, CA, 1994), 233-305, Math. Sci. Res. Inst. Publ. 32, Cambridge Univ. Press, Cambridge, 1997.

[SMA] K. SUIZU, S. MAEDA AND T. ADACHI, Characterization of totally geodesic Kähler immersion, Hokkaido Math. J. 31 (2002), 629-641.

Department OF MATHEMATiCS

NAGOYa Institute OF TECHNOLOGY

GOKISO, NAGOYA, 466-8555

JAPAN

E-mail address: adachi.toshiaki@nitech.ac.jp

DEPARTMENT OF MATHEMATICS

SHIMANE UNIVERSITY

Matsue, Shimane, 690-8504

JAPAN

E-mail address: smaeda@riko.shimane-u.ac.jp
DePaRtMent of Mathematics

SHIMANE UNIVERSITY

Matsue, Shimane, 690-8504

JAPAN

E-mail address: mkimura@ riko.shimane-u.ac.jp 